\title{
miR-1275 inhibits adipogenesis via ELK1 and its expression decreases in obese subjects
}

\author{
Lingxia Pang1,2,", Lianghui You1,", Chenbo Ji1, Chunmei Shi', Ling Chen', Lei Yang1,2, \\ Fangyan Huang1,2, Yahui Zhou'1,2, Jun Zhang', Xiaohui Chen' ${ }^{1}$ and Xirong Guo',2 \\ 1Department of Children Health Care, Nanjing Maternity and Child Health Care Hospital Affiliated to \\ Nanjing Medical University, Nanjing, China \\ Institute of Pediatrics, Nanjing Medical University, Nanjing, China \\ *(L Pang and L You contributed equally to this work)
}

Correspondence should be addressed to $X$ Guo or $X$ Chen Email xrguo@njmu.edu.cn or chenxiaohui327@hotmail.com

\begin{abstract}
Excessive adipocyte differentiation and proliferation are closely associated with the onset of obesity, which has been partially linked to microRNA expression. In previous studies, using miRNA microarray screening, we found that miR-1275 was significantly decreased in human mature adipocytes. In this study, we examined the role of miR-1275 in adipogenesis. Our results indicated that miR-1275 can inhibit the differentiation of human visceral preadipocytes without affecting their proliferation. ELK1, an E-twentysix (ETS)-domain transcription factor associated with adipocyte differentiation, was strongly suppressed by miR-1275 in human visceral adipocytes. This was demonstrated via a dual-luciferase reporter assay and pointed to ELK1 as a direct target of miR-1275. Furthermore, miR-1275 expression was significantly diminished in the visceral adipose tissue of overweight and obese human subjects accompanied by a negative correlation with body mass index. These results suggest that miR-1275 could play a future role in the management of obesity, as a novel therapeutic target or biomarker.
\end{abstract}
Key Words
- miR-1275
- adipogenesis
- ELK1
- obesity

\section{Introduction}

A chronic imbalance between nutrient intake and energy expenditure leads to obesity, which is a major risk factor for a number of metabolic and vascular dysfunctions that include type 2 diabetes, cardiovascular disease, hyperlipidemia, and hypertension (Grundy 2004, Haslam $\&$ James 2005). Since obesity is related to an abnormal increase in the size and/or number of adipocytes, elucidating the detailed mechanisms of adipogenesis is important for comprehending disorders linked with obesity.

Recently, adipogenesis has been shown to be controlled by a tightly regulated transcriptional cascade, where various transcription factors, chromatinremodeling factors, and hormones activate or repress the expression of each other in a sequential manner (Farmer 2006). Among these, peroxisome proliferator-activated receptor gamma (PPARG), a member of the nuclear hormone receptor family, is both necessary and sufficient for adipogenesis (Rosen et al. 1999). No other factor to date has been found to be capable of inducing adipogenesis in the absence of PPARG. CCAAT/enhancer-binding proteins (C/EBPs), including C/EBPA, C/EBPB, and C/EBPD, cooperate with PPARG to induce the expression of many genes important for terminal differentiation (Yeh et al. 1995,

Published by Bioscientifica Ltd 
Lefterova et al. 2008). Many other transcriptional cofactors serve as molecular scaffolds to link the basal transcription machinery with either active or inactive complexes; examples include early adipogenic factors such as Krüppel-like factors (KLFs) (Mori et al. 2005, Oishi et al. 2005), early growth response 20 (KROX20) (Chen et al. 2005), and sterol regulatory element-binding protein 1c (SREBP1c) (Kim et al. 1998), which are activated by adipogenic stimuli. Therefore, understanding the molecular mechanisms of adipogenesis is now seen as a key step against obesity.

MicroRNAs (miRNAs) are another group of adipogenic regulators, and they are becoming an increasingly important target of research into obesity and metabolic syndrome (Esau et al. 2004, Kolfschoten et al. 2009). miRNAs are endogenous noncoding RNAs of 22 nucleotides in length that negatively regulate gene expression at the post-transcriptional level, either by inhibiting translation of the target mRNA or by inducing its degradation. Esau and coworkers (2004) first revealed that human adipocyte differentiation was effectively abolished by the action of an miRNA, miR-143, which reduced both the expression of adipocyte-related genes and triglyceride accumulation. Subsequent studies found that miR-103 (Xie et al. 2009, Trajkovski et al. 2011), miR107 (Trajkovski et al. 2011), and the miR-17-92 cluster (Wang et al. 2008) promoted adipogenic differentiation, whereas LET7 (Sun et al. 2009) and miR-27 (Lin et al. 2009) inhibited adipogenesis. Another study showed differences in miRNA expression between omental and subcutaneous fat in humans (Kloting et al. 2009). More studies on the effect of miRNAs on human adipogenesis are needed, especially those investigating at antiadipogenic miRNAs.

In recent years, human preadipocytes have acted as a useful model in vitro for exploring adipocyte proliferation and differentiation at the molecular level. Our previous studies have profiled differences in miRNA expression across the three main stages of human adipocyte differentiation, using mesenchymal stem cells (hMSCs), visceral preadipocytes (vHPAs), and mature (fully differentiated) adipocytes (unpublished data; details are available upon request). This work indicated a dramatic downregulation of miR-1275 in human mature adipocyte; therefore, we chose this miRNA as the focus of the present study. Expression of miR-1275 has been examined in the context of various physiological processes, but whether it is involved in adipogenesis remains an open question. In this study, we investigate the role of miR-1275 in human visceral adipogenesis.

\section{Materials and methods}

\section{Cell culture and differentiation}

Human visceral preadipocyte (vHPA; purchased from ScienCell Research Laboratories, San Diego, CA, USA) was cultured and maintained in the preadipocyte medium (PAM; purchased from ScienCell Research Laboratories, San Diego, CA, USA) containing 5\% fetal bovine serum (FBS), 1\% preadipocyte growth supplement, and $1 \%$ penicillin/streptomycin at $37^{\circ} \mathrm{C}$ in a humidified atmosphere under $5 \% \mathrm{CO}_{2}$. Differentiation was induced by the incubation of confluent human preadipocytes (day 0) for 4 days in serum-free PAM supplemented with $0.5 \mathrm{mM}$ 3-isobuty-1-methylxanthine (MIX; Sigma), $5 \mu \mathrm{g} / \mathrm{mL}$ insulin (Sigma), $1 \mu \mathrm{M}$ dexamethasone (Sigma), and $1 \mu \mathrm{M}$ rosiglitazone (Sigma). After 4 days, the medium was replaced with serum-free DMEM containing $5 \mu \mathrm{g} / \mathrm{mL}$ insulin, which was changed every 3 days until accumulation of lipid droplets was observed (day 15).

\section{Human subjects}

Abdominal visceral or subcutaneous adipose tissues were prospectively collected from patients undergoing surgery for various disorders. Patients with any type of endocrine disorder, malignancy, or severe systemic illness were not included. The subjects were divided into two groups based on body mass index (BMI): lean subjects $\left(18.5<\mathrm{BMI}<23.9 \mathrm{~kg} / \mathrm{m}^{2}\right.$, visceral $n=12$, subcutaneous $n=6$ ) and overweight and obese subjects (BMI $\geq 24 \mathrm{~kg} / \mathrm{m}^{2}$, visceral $n=12$, subcutaneous $n=5$ ). Biopsies were snapfrozen and stored at $-80^{\circ} \mathrm{C}$ before extraction of RNA. All study participants provided written informed consent, and the experimental protocols were approved by the Ethics Committee of Nanjing Maternity and Child Health Care Hospital Affiliated to Nanjing Medical University, PRC (No. 2011-50, No. 2013-4).

\section{miRNA transduction}

miR-1275 was either overexpressed or knocked down in vHPAs using recombinant lentivirus vectors (GenePharma Co, Ltd, Shanghai, China) containing pre-miR-1275 (miR-1275), negative control precursor miRNA (NC), anti-miRNA against miR-1275 (antimiR-1275), or negative control for the anti-miRNA (anti-NC). For lentiviral infection, cells were plated overnight in six-well plates at a concentration of $3 \times 10^{5}$ cells per well, and were then infected at a multiplicity of infection

Published by Bioscientifica Ltd. 
(MOI) of $2.0 \mu \mathrm{L} /$ well in the presence of Polybrene $(5 \mu \mathrm{g} / \mathrm{mL})$ for $24 \mathrm{~h}$. The medium was replaced on the second day. Green fluorescent protein (GFP) levels were measured at 48 and $72 \mathrm{~h}$, and the lentivirus-mediated expression of miR1275 was verified using quantitative reverse transcription polymerase chain reaction (qRT-PCR).

\section{RNA extraction and qRT-PCR}

RNAs were isolated using an miRNeasy Mini Kit (Qiagen), following the manufacturer's instructions, and the extracted RNA was quantified using a Nanodrop 2.0 instrument (Thermo Fisher Scientific). To quantify miR-1275 expression, total RNA was polyadenylated and reverse-transcribed using a TaqMan MicroRNA Reverse Transcription Kit and TaqMan miRNA assay (Applied Biosystems). U6 small nuclear RNA was used as the internal control. Other qRT-PCR analyses of the markergene expression were performed using Thermo Scientific RevertAid First Strand cDNA Synthesis Kit (Thermo Fisher Scientific) and SYBR Select Master Mix (Applied Biosystems). GAPDH mRNA was used as an internal control. All primers used are listed in Supplementary Table 1, see section on supplementary data given at the end of this article. All qRT-PCR measurements were performed in triplicate using a V7 Fast Real Time PCR system (Applied Biosystems). Relative expression of the genes was calculated and normalized using the $2^{-\Delta \Delta C T}$ method. More details can be found in the supplementary material.

\section{Oil-red-O staining}

Cells were washed twice with PBS, followed by fixation with $4 \%$ paraformaldehyde for $20 \mathrm{~min}$ at room temperature. Then they were washed twice with $60 \%$ isopropanol and then stained with filtered oil-red-O solution (Sigma; stock solution, $3 \mathrm{mg} / \mathrm{mL}$ in isopropanol; working solution, $60 \%$ oil-red-O stock solution and $40 \%$ distilled water) for $30 \mathrm{~min}$ at $37^{\circ} \mathrm{C}$. After staining, cells were washed twice with water for $5 \mathrm{~min}$ to remove unbound dye, observed under a microscope (Axio Observer DI, Zeiss) at 200x magnification, and photographed.

\section{Determination of triglyceride (TG) content}

Human preadipocytes were grown in six-well plates and at approximately 50\% confluence, and then transfected with miR-1275, NC, anti-miR-1275, or anti-NC. Then, they were differentiated into mature adipocytes. At days $4,7,10$, and 15 , the intracellular TG content was determined via a tissue triglyceride assay kit (Lot: \#E1013, Applygen Technologies Inc, Beijing, China), according to the manufacturer's instructions. The absorbance values were measured at a wavelength of $550 \mathrm{~nm}$, normalized to the total protein content, and then expressed as $\mu \mathrm{mol} / \mathrm{g}$ of protein. Blank and calibration wells were analyzed in an identical manner.

\section{Western blot}

Cells were collected in lysis buffer (RIPA; Beyotime, Shanghai, China) supplemented with $1 \mathrm{mM}$ phenylmethanesulfonyl fluoride (PMSF; Beyotime, Shanghai, China), and centrifuged at $14,167 \boldsymbol{g}$ for $15 \mathrm{~min}$. Protein concentrations were determined using a BCA protein assay kit (Thermo Fisher Scientific). The extracts were boiled with $4 \mathrm{X}$ SDS loading buffer $(150 \mathrm{mM}$ Tris- $\mathrm{HCl}$ (pH $6.8), 12 \%$ SDS, $30 \%$ glycerol, $0.02 \%$ bromophenol blue, $6 \% 2$-mercaptoethanol) at $100^{\circ} \mathrm{C}$ for $5 \mathrm{~min}$. Then, $28 \mu \mathrm{g}$ protein were separated on a $10 \%$ SDS-PAGE gel under reduction conditions and electroblotted onto a PVDF membrane (Millipore). The membranes were incubated overnight with primary antibodies against ELK1 (Cat. No. ab32106, Abcam) and GAPDH (Cat. No. AP0063, Bioworlde, Nanjing, China).

\section{DNA plasmid construction and dual-luciferase assay}

Human ELK1 3'-UTR fragments containing potential binding sites for miR-1275 were amplified by PCR from human genomic DNA with XhoI and NotI linkers. The fragments were cloned into a psi-CHECK2 vector (Promega) downstream of the luciferase gene to generate the recombinant vectors psi-CHECK2-ELK1-WT (W.T. vector). Psi-CHECK2-ELK1-MUT (MUT. vector) was mutated by mutagenesis PCR using their wild-type vector as the template. For the luciferase reporter assay, HEK293T cells were transiently cotransfected with mimics (miRNA-1275 mimics or control mimics (CM; RiboBio Co, Ltd, Guangzhou, China) and reporter vectors (W.T., MUT, or control vector), using Lipofectamine 3000 (Invitrogen). Luciferase activities were measured using a dual-luciferase reporter assay system (Promega) according to the manufacturer's instructions at $48 \mathrm{~h}$ posttransfection. Renilla luciferase activity was normalized to the corresponding firefly luciferase activity and plotted as a percentage of the control (cells cotransfected with the corresponding concentration of control mimics). All experiments were performed in triplicate wells and repeated at least three times $(n \geq 3)$.

Published by Bioscientifica Ltd 

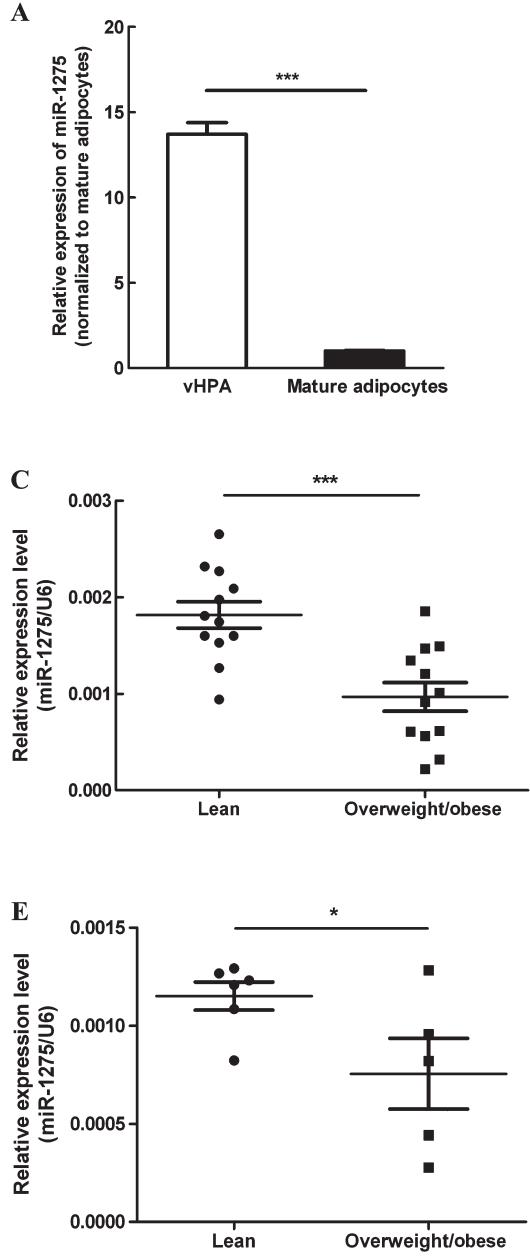
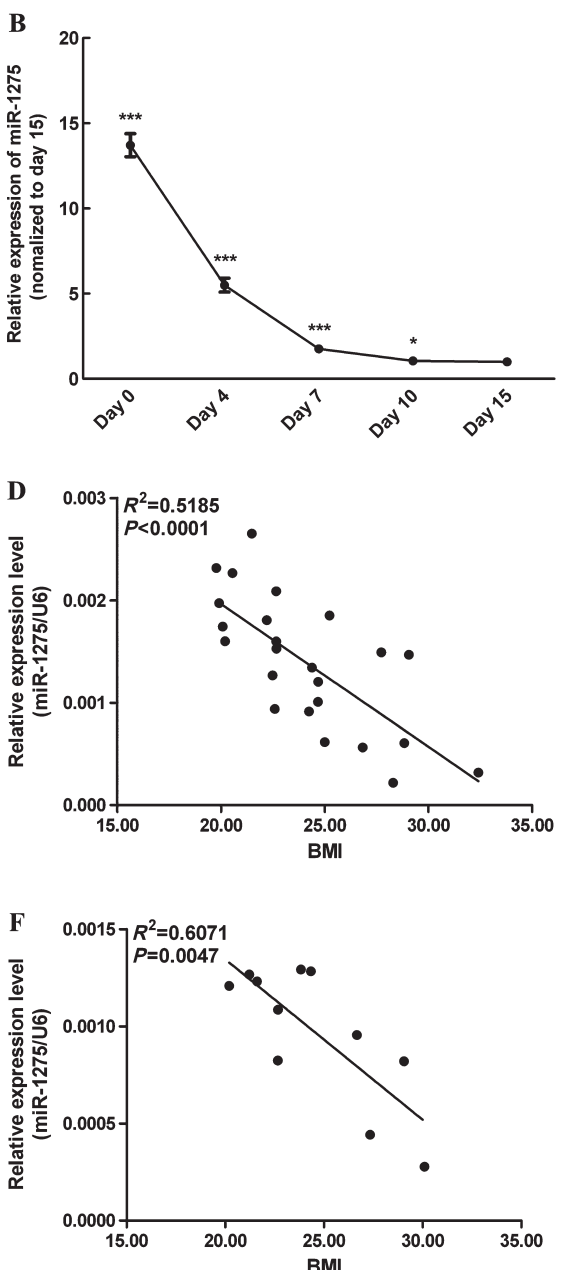

Figure 1

miR-1275 is differentially expressed during human adipogenesis and reduced in the visceral adipose tissue of overweight and obese subjects.

(A) miR-1275 levels were measured in human visceral preadipocytes (vHPAs) between day 0 and 15 of differentiation $(n=3)$. (B) Expression of miR-1275 over a 15-day period of adipocyte differentiation $(n=3)$; miR-1275 levels were normalized to U6 RNA and then expressed relative to the day-15 value. ( $C$ and $E$ ) miR-1275 expression, as determined by qRT-PCR, was downregulated in visceral (C) or subcutaneous (E) adipose tissue from overweight and obese human subjects versus lean subjects. ( $D$ and $F$ ) Linear regression analysis indicated a negative relationship between visceral (D) or subcutaneous (F) adipose tissue miR-1275 levels and body mass index (BMI). Data are expressed as the mean \pm S.D. $\star P<0.05, * * * P<0.001$

\section{Statistical analysis}

The data were analyzed using SPSS 17.0 program. All values are presented as the mean \pm s.E.M. or SD of at least three independent experiments $(n \geq 3)$. A two-tailed Student's $t$-test was used to determine the statistical significance of differences between experimental groups; $P<0.05$ was considered to be statistically significant.

Table 1 General and biochemical characteristics of the study subjects in visceral adipose tissues.

\begin{tabular}{|c|c|c|}
\hline \multirow[b]{2}{*}{ Parameters } & \multicolumn{2}{|c|}{ Visceral adipose tissues } \\
\hline & $\begin{array}{l}\text { Lean } \\
(n=12)\end{array}$ & $\begin{array}{l}\text { Overweight/obese } \\
\qquad(n=12)\end{array}$ \\
\hline Age (years) & $41.92 \pm 0.86$ & $48.67 \pm 0.54$ \\
\hline Gender (female/male) & $11 / 1$ & $10 / 2$ \\
\hline BMI $\left(\mathrm{kg} / \mathrm{m}^{2}\right)$ & $21.44 \pm 0.10$ & $26.78 \pm 0.21 * * *$ \\
\hline Total cholesterol (mmol/L) & $4.03 \pm 0.14$ & $4.04 \pm 0.16$ \\
\hline Triacylglycerol (mmol/L) & $1.15 \pm 0.06$ & $2.64 \pm 0.15^{*}$ \\
\hline Glucose (mmol/L) & $4.94 \pm 0.03$ & $5.12 \pm 0.03$ \\
\hline
\end{tabular}

Data are expressed as mean \pm S.E.M. ${ }^{*} P<0.05, * * * P<0.001$

\section{Results}

miR-1275 is highly expressed in human preadipocytes and its expression are reduced in visceral adipose tissues from overweight and obese subjects

To identify the miRNAs related to adipogenesis, the miRNA expression of cells at the early, middle, and late stages of

Table 2 General and biochemical characteristics of the study subjects in subcutaneous adipose tissues.

\begin{tabular}{|c|c|c|}
\hline \multirow[b]{2}{*}{ Parameters } & \multicolumn{2}{|c|}{ Subcutaneous adipose tissues } \\
\hline & $\begin{array}{l}\text { Lean } \\
(n=6)\end{array}$ & $\begin{array}{l}\text { Overweight/obese } \\
\qquad(n=5)\end{array}$ \\
\hline Age (years) & $53.83 \pm 1.89$ & $52.00 \pm 4.33$ \\
\hline Gender (female/male) & $5 / 1$ & $5 / 0$ \\
\hline BMI $\left(\mathrm{kg} / \mathrm{m}^{2}\right)$ & $22.03 \pm 0.21$ & $27.50 \pm 0.74 * * *$ \\
\hline Total cholesterol (mmol/L) & $4.14 \pm 0.12$ & $2.54 \pm 0.42 *$ \\
\hline Triacylglycerol (mmol/L) & $1.03 \pm 0.04$ & $4.02 \pm 0.73 * *$ \\
\hline
\end{tabular}

Data are expressed as mean \pm S.E.M. ${ }^{*} P<0.05,{ }^{*} P<0.01, * * * P<0.001$

Published by Bioscientifica Ltd. 
A
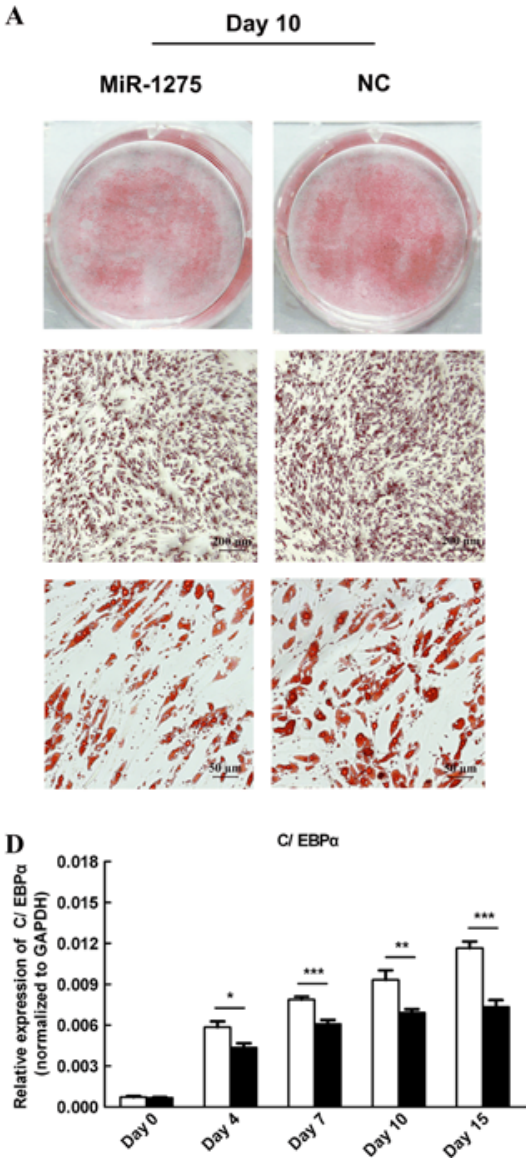

B

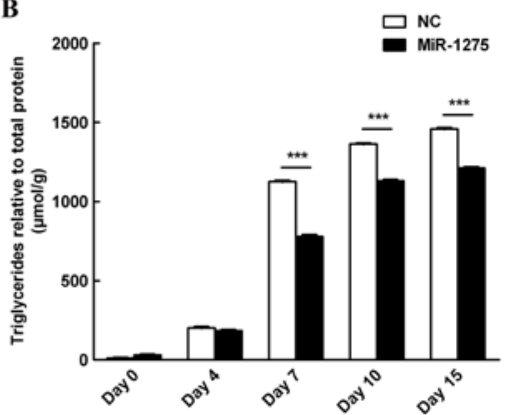

C

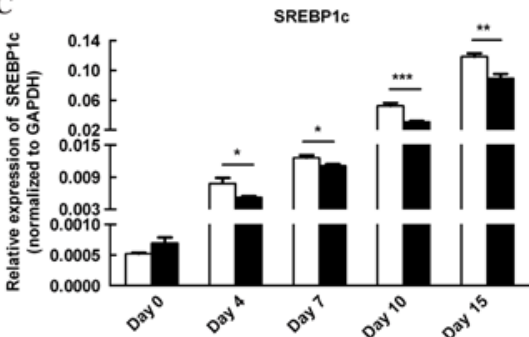

E

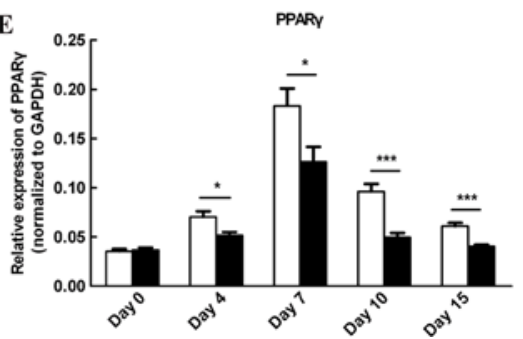

Figure 2

Overexpression of miR-1275 inhibits human preadipocyte differentiation. (A) Representative images of cells stained with oil red $O$ on day 15 of differentiation showing the effect of miR-1275 overexpression. (B) Triglyceride accumulation was quantified at different time points of adipogenesis $(n=3)$. (C, D and E) The relative mRNA levels of adipocyte-specific genes were assessed by qRT-PCR at days $0,4,7,10$, and 15 . The data represent the mean \pm S.E.M. of three independent experiments. ${ }^{*} P<0.05, * * P<0.01$, $* * * P<0.001$. PPARG, peroxisome proliferatoractivated receptor gamma; C/EBPA, CCAAT/ enhancer-binding protein alpha; SREBP1C, sterol regulatory element-binding protein $1 c$. adipogenesis (hMSCs, vHPAs, and mature adipocytes) was analyzed using miRNA microarrays. We recognized that miR-1275 levels were significantly decreased 14.3-fold in mature adipocytes, and then used qRT-PCR to compare miR-1275 expression in vHPAs and mature adipocytes. We found that miR-1275 expression decreased approximately 14-fold over the 15 days of adipogenesis (Fig. 1A), with most of the decline occurring in the first 7 days (Fig. 1B), further suggesting that miR-1275 is highly expressed in preadipocytes (vHPAs) and may be linked to adipocyte differentiation.

To examine the relationship between miR-1275 and obesity, we measured miR-1275 levels in human adipose tissue of overweight and obese human subjects and compared them with those of matched lean subjects. All the adipose-tissue samples were obtained from the greater omentum or subcutaneous adipose tissue of patients undergoing surgery for abdominal disorders, excluding endocrine disorders, malignancy, and severe systemic illness (Tables 1 and 2). We observed that the miR-1275 expression was significantly decreased in visceral adipose tissue of the overweight and obese patients, compared with those in the lean group (Fig. 1C). There also was a significant negative correlation between miR-1275 and BMI (Fig. 1D; $R^{2}=0.5185, P<0.0001$, Pearson's correlation). Additionally, an obvious reduction in miR-1275 level (Fig. 1E) as well as a negative correlation between miR-1275 and BMI (Fig. 1F; $R^{2}=0.6071, P=0.0047$, Pearson's correlation) appeared in subcutaneous adipose tissue. These data suggest that miR-1275 could be used as a biomarker for screening the possibility to become obese.

\section{miR-1275 does not influence proliferation of human adipocytes}

Adipogenesis involves cell proliferation and differentiation, both of which have been shown to be regulated by miRNA (Wang et al. 2008). We transduced vHPAs with miR-1275, and after $72 \mathrm{~h}, 90 \%$ of the cells were found to be GFP-positive by fluorescence microscopy, and no morphological differences were observed between the transduced and the control vHPAs (Supplementary Fig. S1A). Using qRT-PCR, the abundance of miR-1275 was demonstrated by the fact that miR-1275 expression was elevated in transduced cells, when compared with NC-infected cells (Supplementary Fig. S1B).

Published by Bioscientifica Ltd. 


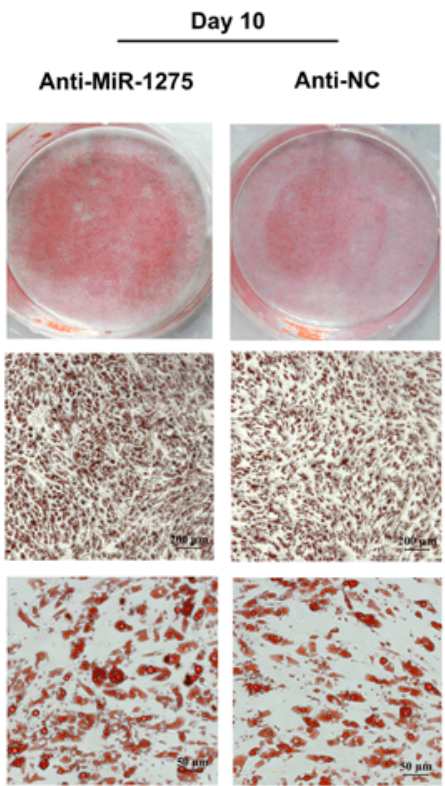

D

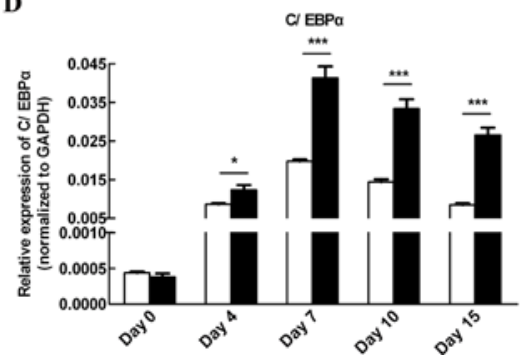

B

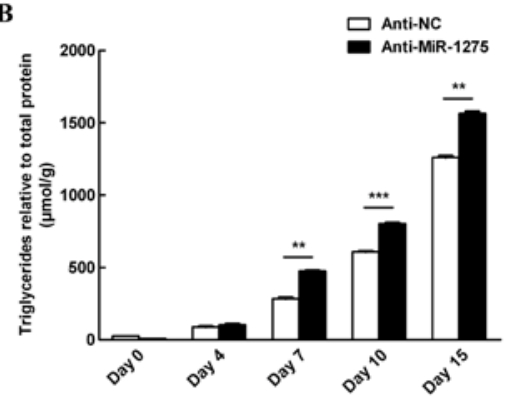

C

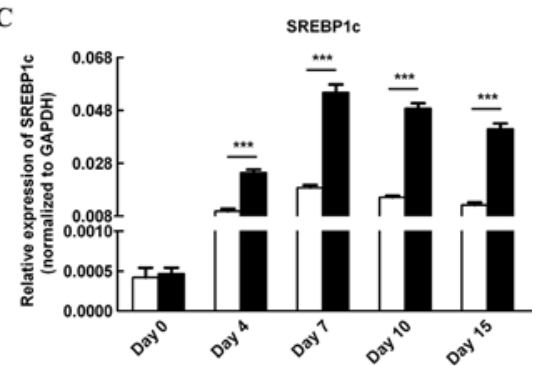

E

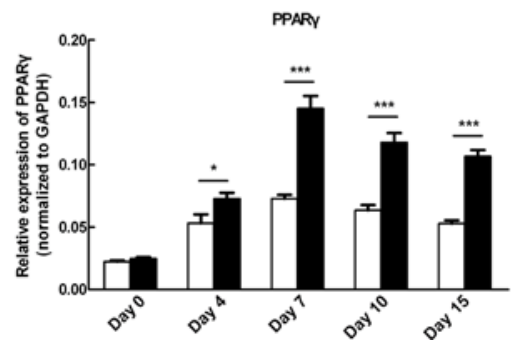

Figure 3

Downregulation of miR-1275 promotes human preadipocyte differentiation. (A) Cellular lipid accumulation was detected by oil-red-O staining at day 15. (B) Triglyceride levels were analyzed at days $4,7,10$, and $15(n=3)$. (C, D and E) mRNA expression of adipocyte-specific genes was estimated at days 4, 7, 10, and 15. The data represent the mean \pm S.E.M. of three independent experiments. ${ }^{*} P<0.05, * * P<0.01, * * * P<0.001$.
In order to determine whether miR-1275 expression is involved in the proliferation of visceral preadipocytes, we measured the numbers of cultured cells using an MTT assay. As depicted in Supplementary Fig. S1C, there was no obvious difference in absorbance at $490 \mathrm{~nm}$ between the miR-1275 overexpression group and the negative-control group after $48 \mathrm{~h}$. Furthermore, the effects of miR-1275 knockdown (anti-miR-1275) on proliferation were not significantly different, consistent with those of miR-1275 overexpression (Supplementary Fig. S1D). These results indicate that the miR-1275 expression has no functional significance in human preadipocyte proliferation.

\section{miR-1275 suppresses human adipocyte differentiation}

Given these observations, we hypothesized that miR-1275 has a functional role in human adipocyte differentiation. To test this hypothesis, vHPAs were transduced with either miR-1275 or the negative control, NC. When adipogenesis was hormonally stimulated in these cells, it was found to be significantly reduced in those overexpressing miR-1275, as represented by morphological changes observed after oil-red-O staining (Fig. 2A). The suppressed differentiation was consistent with the reduction in triglyceride content, also observed (Fig. 2B). Furthermore, these results were accompanied by decreases in the mRNA levels of some key adipogenic transcription factors. As shown in Fig. 2C, $\mathrm{D}$ and $\mathrm{E}$, the expression of PPARG, C/EBPA, and SREBP1C significantly dropped in the cells overexpressing miR-1275 at 7,10 , and 15 days after the induction of adipogenic differentiation.

However, knockdown of miR-1275 strongly accelerated adipogenesis in human visceral preadipocytes, as indicated by oil-red-O staining (Fig. 3A) and the TG assay (Fig. 3B). QRT-PCR analysis indicated that the expression of the adipocyte-specific factors (PPARG, $C / E B P A$, and $S R E B P 1 C$ ) was elevated at different time points during adipogenesis (Fig. 3C, D and E). http://jme.endocrinology-journals.org DOI: 10.1530/JME-16-0007
() 2016 Society for Endocrinology Printed in Great Britain 


\section{miR-1275 downregulates ELK1 via binding to its 3'-UTR region}

Suppression of gene expression by miRNAs is often via imperfect base pairing to the 3 '-untranslated region (3'-UTR) of their targetmRNAs. To investigate the mechanism by which miR-1275 inhibits human preadipocyte differentiation, we sought to identify potential 3'-UTR targets. Bioinformatic analysis, using miRBase, TargetScan, miRDB, and miRanda, revealed four potential miR-1275 binding sites in the 3 '-UTR of ELK1, and the binding sequences were conserved among eight species. Previous reports have demonstrated the importance of ELK1 in adipocyte differentiation (Wang et al. 2009, Ge et al. 2011, Lin et al. 2014).
To examine whether miR-1275 affects ELK1 expression, we used qRT-PCR to measure ELK1 mRNA in cultured preadipocytes, with either up- or downregulated miR-1275, at different time points during adipocyte differentiation. The results showed a negative relationship (Fig. 4A and C). Then, we assessed ELK1 protein expression via Western blot, and found that miR-1275 inhibited ELK1 (Fig. 4B and D), which is consistent with the hypothesis that ELK1 is a direct target of miR-1275. In addition, the expression level of ELK1 was increased in visceral adipose tissue of overweight and obese human subjects (Fig. 4G).

To verify miR-1275 binding, we generated a luciferase reporter vector containing either the wild-type 3'-UTR of ELK1 or a variant $3^{\prime}$-UTR with a mutated binding

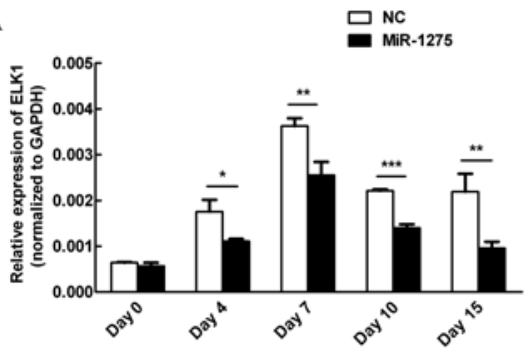

C

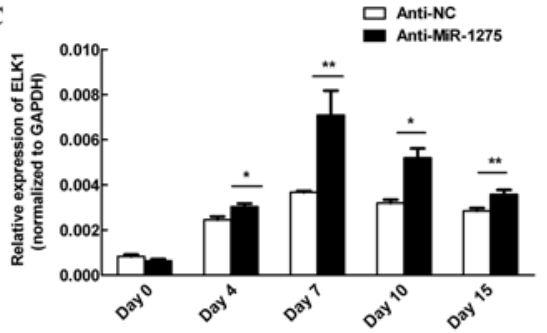

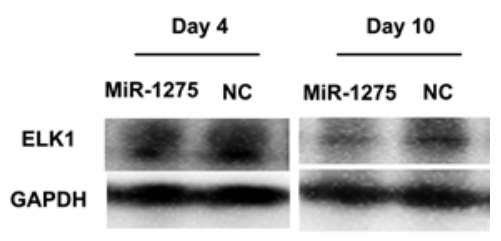

D

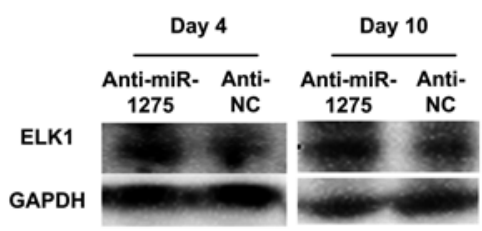

Position 332-338 of W.T. ELK1 3.UTR Position 214-220 of W.T. ELK1 3'UTR Position 937-943 of W.T. ELK1 3'UTR Position 1000-1006 of W.T. ELK1 3UTR

MUT. ELK1 3'UTR

Hsa-miR-1275

F

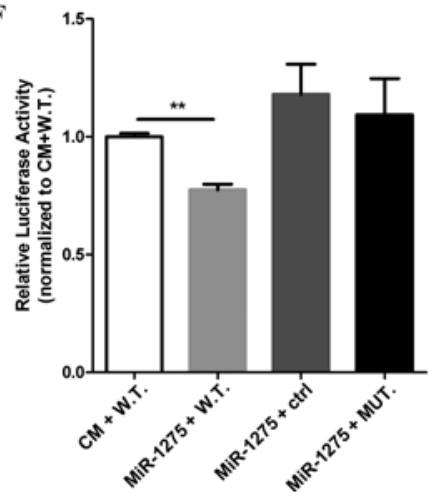

CUGUCGGAGAGGGGGUG

I|I| II I III

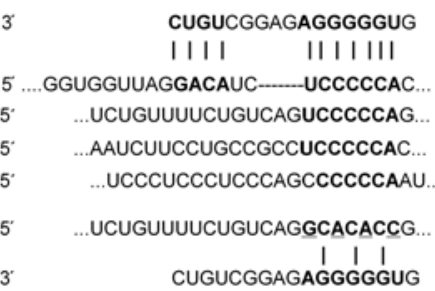

G

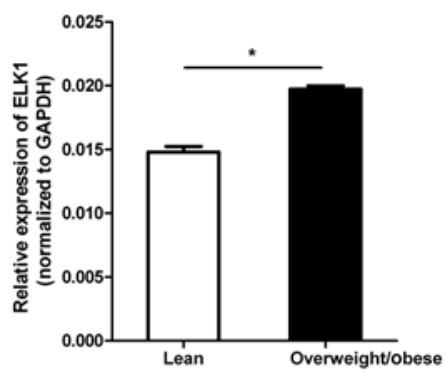

Figure 4

miR-1275 negatively regulates ELK1 in human visceral adipocytes (vHPAs). ELK1 mRNA levels in preadipocytes transduced with miR-1275 were analyzed by qRT-PCR during adipogenesis at days $0,4,7,10$, and $15(\mathrm{~A})$ and ELK1 protein was measured by Western blot at day 7 (B). (C and D) VHPAs infected with anti-miR-1275 were analyzed by qRT-PCR and Western blot for ELK1 levels at the indicated time points. (E) miR-1275 binding sequences of the wild-type (W.T. ELK1 3'-UTR) and mutant (MUT. ELK1 3'-UTR) reporter vectors. The "seed" match region of miR-1275 is highlighted in bold and the mutant sites are underlined.

(F) HEK293T cells were transiently transfected with an miR-1275 mimic or a negative control mimic (CM) combined with a psi-CHECK2 control plasmid (a modified psi-CHECK2 plasmid containing the wild-type ELK1 3'-UTR), or an ELK1 3'-UTR with a mutated binding sequence. Luciferase activity was analyzed $48 \mathrm{~h}$ later; for each sample, renilla luciferase activity was normalized to firefly luciferase $(n=6)$. (G) The expression of ELK1 was upregulated in visceral adipose tissue of overweight and obese human subjects. Data are presented as the mean \pm S.E.M. of three independent experiments. ${ }^{*} P<0.05, * * P<0.01$. http://jme.endocrinology-journals.org DOI: 10.1530/JME-16-0007
C 2016 Society for Endocrinology Printed in Great Britain
Published by Bioscientifica Ltd. 
A

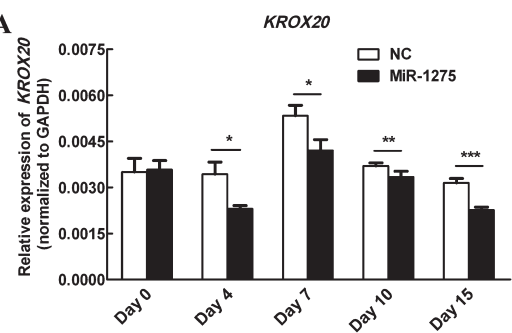

C

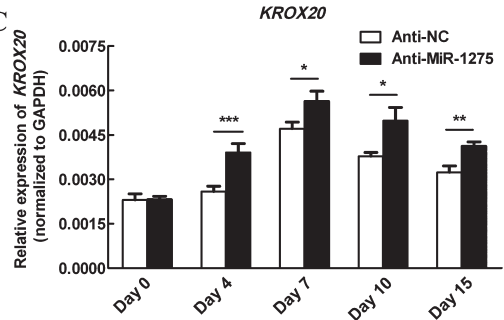

$\mathbf{E}$

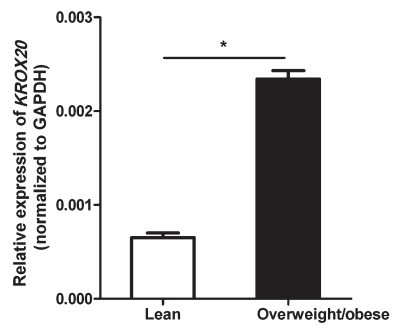

B

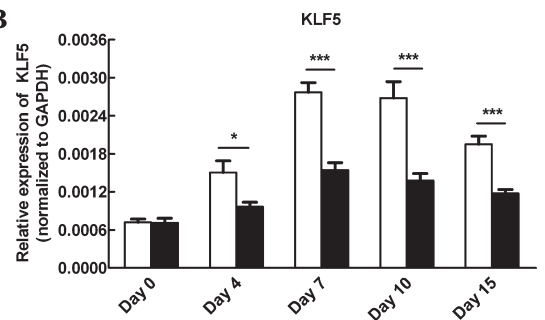

D

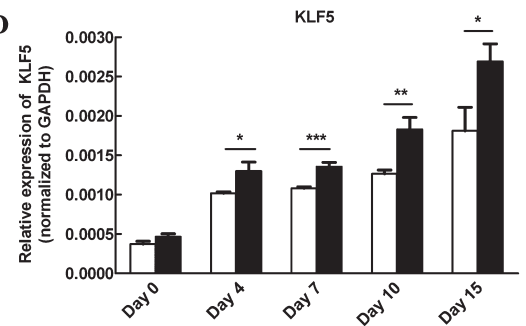

F

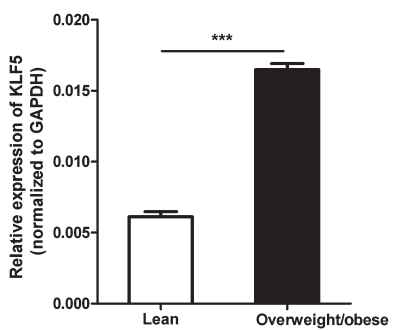

Figure 5

miR-1275 regulates the expression of $K R O X 20$ and KLF5. KROX20 and KLF5 mRNA levels were analyzed by qRT-PCR during adipogenesis at days $0,4,7,10$, and 15 in preadipocytes overexpressing miR-1275 (A and B) and in miR-1275 knockdowns (C and D). (E and F) KROX20 and KLF5 expressions were upregulated in visceral adipose tissue from overweight and obese human subjects. Data are presented as the mean \pm S.E.M. of three independent experiments. ${ }^{*} P<0.05, * * P<0.01$, $* * * P<0.001$. KROX20, early growth response 2 ; KLF5, Krüppel-like factor 5 . sequence. A dual-luciferase assay (Fig. 4E and F) confirmed that miR-1275 directly bound to the 3'-UTR of ELK1.

\section{miR-1275 regulates the expression of KROX20 and KLF5}

A previous report (Wang et al. 2009) found that ELK1 participated in the adipogenic transcription cascade by altering the expression of KLF5 and KROX20; therefore, we measured their mRNA expression during differentiation of adipocytes with either up- or downregulated miR-1275. We found that KROX2O and KLF5 expression was reduced in the overexpressing cells (Fig. 5A and B), and increased in the knockdown cells (Fig. 5C and D). Moreover, KROX2O and KLF5 expression were upregulated in visceral adipose tissue from overweight and obese human subjects vs lean subjects. These results indicate that KLF5 and KROX2O may be involved in miR-1275-mediated regulation of human adipocyte differentiation.

\section{Discussion}

Visceral obesity is the huge burden on the welfare of society, thus fanning interest in studies about the mechanisms of adipocyte differentiation to decrease obesity prevalence and mitigate its detrimental physiological effects. Recently, studies on the functional role of miRNAs in adipogenesis have mushroomed. miR-143 (Esau et al. 2004, Oskowitz et al. 2008), miR-8 (Kennell et al. 2008), miR-21 (Kim et al. 2009), and miR-378 (Carrer et al. 2012) have been reported to enhance adipogenesis, whereas the investigations of miRNAs in suppressing adipogenesis are relatively less.

In previous studies aimed at achieving better understanding of the part played by miRNAs in human adipogenesis, we found that miR-148a (Shi et al. 2015), miR-146b (Chen et al. 2014), and miR-26b (Song et al. 2014) positively regulate adipogenesis. In this study, we identified miR-1275 as a novel negative regulator of human visceral preadipocyte differentiation. miR-1275 is an intergenic miRNA, which is encoded by chromosome 6. Previous reports suggested that miR-1275 is associated with cancer (Ozata et al. 2011, Agrawal et al. 2014), coronary heart disease (Huan et al. 2015), neural tube defects in fetuses (Gu et al. 2012), and mitochondrial dysfunction (Nymark et al. 2015). Katsushima and coworkers (2012) found that the inhibition of miR-1275 induces the expression of oligodendroglial-lineage proteins and suppresses tumor-cell proliferation in human glioma stem-like cells. However, to date, there are no reports indicating that

Published by Bioscientifica Ltd. 
miR-1275 is involved in adipogenesis. The overexpression observed in our study and the results from knockdown experiments suggest that miR-1275 blocks adipocyte differentiation without influencing cell proliferation.

Our results indicated that miR-1275 directly bound to the 3'-UTR of ELK1 (Supplementary Fig. S1A). ELK1, as a member of the ternary complex factor (TCF) subfamily of E-twenty-six (ETS)-domain transcription factors, is regulated by three major MAP kinase pathways and the CaMKII pathway (Yordy \& Muise-Helmericks 2000). Being a nuclear transcription factor, phosphorylation of ELK1 will activate many other transcription factors, including immediate early genes (Yang et al. 1999). ELK1 has been found to modulate many developmental processes such as cellular proliferation (Hasan \& Schafer 2008), neuronal differentiation (Vanhoutte et al. 2001), and tumorigenesis (Chai et al. 2001). Recently, Ge and coworkers (2011) showed that ELK1 plays a key role in adipogenesis. They found that mediator MED23 links insulin signaling to the adipogenesis transcription cascade, which includes ELK1 (Ge et al. 2011). In this study, we found that ELK1 always kept a negative relationship with miR-1275 at different time points during adipocyte differentiation in either up- or downregulated experiments (both at mRNA and protein levels) (Fig. 4A, B, C and D), and ELK1 expression was increased in overweight and obese human subjects (Fig. 4G). Furthermore, it was demonstrated that ELK1 was a direct target of the endogenous miR-1275 via a dualluciferase reporter assay (Fig. 4F).

We also found that miR-1275 expression was inversely related to the mRNA levels of KROX20 and KLF5 both in adipocytes (Fig. 5A, B, C and D) and visceral adipose tissue (Fig. 5E and F). Previous reports have demonstrated that KROX20 is a member of the EGR family of zinc-finger transcription factors, and it is required in the early phase of adipocyte differentiation (Chen et al. 2005). KLF5 is one of the seven KLF proteins known to be associated with adipocyte differentiation (Oishi et al. 2005). A recent report revealed that ELK1 phosphorylation by MED23/ELK1 connects the insulin signaling pathway to the adipogenic transcription cascade by controlling insulin-MAPK-stimulated expression of KROX20 (Wang et al. 2009), following changes in the basal level of KLF5. In our study, we detected an increased expression of KLF5 and KROX20 (Fig. 5C and D), while ELK1 expression level increased (Fig. 4C and D) in loss-of-function experiments. Moreover, we found a decreased expression of KLF5 and KROX20 (Fig. 5A and B), while ELK1 expression level declined (Fig. 4A and B) in gain-of-function experiments. Therefore, we believed that the ELK1 expression correlates positivity with KLF5 and KROX20. Further studies are required to confirm whether miR-1275 induces ELK1 to promote adipogenesis through the insulin-MAPK-ELK1-MED23KROX20 pathway.

Finally, we investigated whether miR-1275 expression in adipose tissue varied according to obesity, and observed downregulation in overweight and obese human subjects, a result that is consistent with the in vitro changes observed in vHPAs. Although it seems that there are differences in the mean age between the lean group and the overweight group as given in Table 1. Because the large age span of the two groups, the SDs are relatively large. And we did not get a significant difference between two groups by t-test. Furthermore, miR-1275 has a significant negative correlation with BMI in visceral adipose tissue as well as in subcutaneous adipose tissue.

In summary, we have shown that miR-1275 is a novel negative regulator of human visceral preadipocyte differentiation, which appears to act via posttranscriptional silencing of ELK1. miR-1275 declined dramatically as human adipocytes differentiated, and gain- and loss-of-function experiments in human preadipocytes indicated that miR-1275 inhibited adipogenesis. Moreover, miR-1275 expression was found to be downregulated in the adipose tissue of overweight and obese patients, when compared with lean patients. Thus, miR-1275 is a potential biomarker for screening the possibility to become obese, and may be an attractive target for new therapies against obesity and metabolic syndrome.

\section{Supplementary data}

This is linked to the online version of the paper at http://dx.doi.org/10.1530/ JME-16-0007

Declaration of interest

The authors declare that there is no conflict of interest that could be perceived as prejudicing the impartiality of the research reported.

\section{Funding}

This study was supported by Grants from the National Key Basic Research Program of China (2013CB530604), the Key Project of National Natural Science Foundation of China (81330067), the National Natural Science Foundation of China (81300706), the Natural Science Foundation of Jiangsu Province China (BK20130075), and the Talent Work Leading Group of Jiangsu Province (333 High-level Talents Training Project, No. 201104013).

Published by Bioscientifica Ltc. 


\section{References}

Agrawal R, Pandey P, Jha P, Dwivedi V, Sarkar C \& Kulshreshtha R 2014 Hypoxic signature of microRNAs in glioblastoma: insights from small RNA deep sequencing. BMC Genomics 15686. (doi:10.1186/1471-2164-15-686)

Carrer M, Liu N, Grueter CE, Williams AH, Frisard MI, Hulver MW, Bassel-Duby R \& Olson EN 2012 Control of mitochondrial metabolism and systemic energy homeostasis by microRNAs 378 and 378*. PNAS 109 15330-15335. (doi:10.1073/pnas.1207605109)

Chai Y, Chipitsyna G, Cui J, Liao B, Liu S, Aysola K, Yezdani M, Reddy ES \& Rao VN 2001 c-Fos oncogene regulator Elk-1 interacts with BRCA1 splice variants BRCA1a/1b and enhances BRCA1a/1bmediated growth suppression in breast cancer cells. Oncogene 20 1357-1367. (doi:10.1038/sj.onc.1204256)

Chen Z, Torrens JI, Anand A, Spiegelman BM \& Friedman JM 2005 Krox20 stimulates adipogenesis via C/EBPbeta-dependent and -independent mechanisms. Cell Metabolism 1 93-106. (doi:10.1016/j. cmet.2004.12.009)

Chen L, Dai YM, Ji CB, Yang L, Shi CM, Xu GF, Pang LX, Huang FY, Zhang CM \& Guo XR 2014 MiR-146b is a regulator of human visceral preadipocyte proliferation and differentiation and its expression is altered in human obesity. Molecular and Cellular Endocrinology 393 65-74. (doi:10.1016/j.mce.2014.05.022)

Esau C, Kang X, Peralta E, Hanson E, Marcusson EG, Ravichandran LV, Sun Y, Koo S, Perera RJ, Jain R, et al. 2004 MicroRNA-143 regulates adipocyte differentiation. Journal of Biological Chemistry 279 5236152365. (doi:10.1074/jbc.C400438200)

Farmer SR 2006 Transcriptional control of adipocyte formation. Cell Metabolism 4 263-273. (doi:10.1016/j.cmet.2006.07.001)

Ge X, Chen C, Hui X, Wang Y, Lam KS \& Xu A 2011 Fibroblast growth factor 21 induces glucose transporter-1 expression through activation of the serum response factor/Ets-like protein-1 in adipocytes. Journal of Biological Chemistry 286 34533-34541. (doi:10.1074/jbc.M111.248591)

Grundy SM 2004 Obesity, metabolic syndrome, and cardiovascular disease. Journal of Clinical Endocrinology \& Metabolism 89 2595-2600. (doi:10.1210/jc.2004-0372).

Gu H, Li H, Zhang L, Luan H, Huang T, Wang L, Fan Y, Zhang Y, Liu X, Wang W, et al. 2012 Diagnostic role of microRNA expression profile in the serum of pregnant women with fetuses with neural tube defects. Journal of Neurochemistry 122 641-649. (doi:10.1111/ j.1471-4159.2012.07812.x)

Hasan RN \& Schafer AI 2008 Hemin upregulates Egr-1 expression in vascular smooth muscle cells via reactive oxygen species ERK-1/2Elk-1 and NF-kappaB. Circulation Research 102 42-50. (doi:10.1161/ CIRCRESAHA.107.155143)

Haslam DW \& James WP 2005 Obesity. Lancet 366 1197-1209. (doi:10.1016/S0140-6736(05)67483-1)

Huan T, Rong J, Tanriverdi K, Meng Q, Bhattacharya A, McManus DD, Joehanes R, Assimes TL, McPherson R, Samani NJ, et al. 2015 Dissecting the roles of microRNAs in coronary heart disease via integrative genomic analyses. Arteriosclerosis, Thrombosis, and Vascular Biology 35 1011-1021. (doi:10.1161/ATVBAHA.114.305176)

Katsushima K, Shinjo K, Natsume A, Ohka F, Fujii M, Osada H, Sekido Y \& Kondo Y 2012 Contribution of microRNA-1275 to Claudin11 protein suppression via a polycomb-mediated silencing mechanism in human glioma stem-like cells. Journal of Biological Chemistry $\mathbf{2 8 7}$ 27396-27406. (doi:10.1074/jbc.M112.359109)

Kennell JA, Gerin I, MacDougald OA \& Cadigan KM 2008 The microRNA miR-8 is a conserved negative regulator of Wnt signaling. PNAS 105 15417-15422. (doi:10.1073/pnas.0807763105)

Kim JB, Wright HM, Wright M \& Spiegelman BM 1998 ADD1/SREBP1 activates PPARgamma through the production of endogenous ligand. PNAS 95 4333-4337. (doi:10.1073/pnas.95.8.4333)

Kim YJ, Hwang SJ, Bae YC \& Jung JS 2009 MiR-21 regulates adipogenic differentiation through the modulation of TGF-beta signaling in mesenchymal stem cells derived from human adipose tissue. Stem Cells 27 3093-3102. (doi:10.1002/stem.235)

Kloting N, Berthold S, Kovacs P, Schon MR, Fasshauer M, Ruschke K, Stumvoll M \& Bluher M 2009 MicroRNA expression in human omental and subcutaneous adipose tissue. PLOS ONE 4 e4699. (doi:10.1371/journal.pone.0004699)

Kolfschoten IG, Roggli E, Nesca V \& Regazzi R 2009 Role and therapeutic potential of microRNAs in diabetes. Diabetes, Obesity and Metabolism 11 (Supplement 4) 118-129. (doi:10.1111/ j.1463-1326.2009.01118.x)

Lefterova MI, Zhang Y, Steger DJ, Schupp M, Schug J, Cristancho A, Feng D, Zhuo D, Stoeckert CJ Jr, Liu XS, et al. 2008 PPARgamma and $\mathrm{C} / \mathrm{EBP}$ factors orchestrate adipocyte biology via adjacent binding on a genome-wide scale. Genes \& Development 22 2941-2952. (doi:10.1101/gad.1709008)

Lin Q, Gao Z, Alarcon RM, Ye J \& Yun Z 2009 A role of miR-27 in the regulation of adipogenesis. FEBS Journal 276 2348-2358. (doi:10.1111/j.1742-4658.2009.06967.x)

Lin X, Li G, He X, Ma X, Zhang K, Zhang H, Zeng G \& Wang Z 2014 FGF21 inhibits apolipoprotein(a) expression in HepG2 cells via the FGFR1-ERK1/2-Elk-1 pathway. Molecular and Cellular Biochemistry 393 33-42. (doi:10.1007/s11010-014-2044-0)

Mori T, Sakaue H, Iguchi H, Gomi H, Okada Y, Takashima Y, Nakamura K, Nakamura T, Yamauchi T, Kubota N, et al. 2005 Role of Kruppel-like factor 15 (KLF15) in transcriptional regulation of adipogenesis. Journal of Biological Chemistry 280 12867-12875. (doi:10.1074/jbc.M410515200)

Nymark P, Wijshoff P, Cavill R, van Herwijnen M, Coonen ML, Claessen S, Catalan J, Norppa H, Kleinjans JC \& Briede JJ 2015 Extensive temporal transcriptome and microRNA analyses identify molecular mechanisms underlying mitochondrial dysfunction induced by multi-walled carbon nanotubes in human lung cells. Nanotoxicology 9 624-635. (doi:10.3109/17435390.2015.1017022)

Oishi Y, Manabe I, Tobe K, Tsushima K, Shindo T, Fujiu K, Nishimura G, Maemura K, Yamauchi T, Kubota N, et al. 2005 Kruppel-like transcription factor KLF5 is a key regulator of adipocyte differentiation. Cell Metabolism 1 27-39. (doi:10.1016/j. cmet.2004.11.005)

Oskowitz AZ, Lu J, Penfornis P, Ylostalo J, McBride J, Flemington EK, Prockop DJ \& Pochampally R 2008 Human multipotent stromal cells from bone marrow and microRNA: regulation of differentiation and leukemia inhibitory factor expression. PNAS 105 18372-18377. (doi:10.1073/pnas.0809807105)

Ozata DM, Caramuta S, Velazquez-Fernandez D, Akcakaya P, Xie H, Hoog A, Zedenius J, Backdahl M, Larsson C \& Lui WO 2011 The role of microRNA deregulation in the pathogenesis of adrenocortical carcinoma. Endocrine-Related Cancer 18 643-655. (doi:10.1530/ERC11-0082)

Rosen ED, Sarraf P, Troy AE, Bradwin G, Moore K, Milstone DS, Spiegelman BM \& Mortensen RM 1999 PPAR gamma is required for the differentiation of adipose tissue in vivo and in vitro. Molecular Cell 4 611-617. (doi:10.1016/S1097-2765(00)80211-7)

Shi C, Zhang M, Tong M, Yang L, Pang L, Chen L, Xu G, Chi X, Hong Q, Ni Y, et al. 2015 miR-148a is associated with obesity and modulates adipocyte differentiation of mesenchymal stem cells through Wnt signaling. Scientific Reports 5 9930. (doi:10.1038/ srep09930)

Song G, Xu G, Ji C, Shi C, Shen Y, Chen L, Zhu L, Yang L, Zhao Y \& Guo X 2014 The role of microRNA-26b in human adipocyte differentiation and proliferation. Gene 533 481-487. (doi:10.1016/j. gene.2013.10.011)

Sun T, Fu M, Bookout AL, Kliewer SA \& Mangelsdorf DJ 2009 MicroRNA let-7 regulates 3T3-L1 adipogenesis. Molecular Endocrinology 23 925931. (doi:10.1210/me.2008-0298)

Trajkovski M, Hausser J, Soutschek J, Bhat B, Akin A, Zavolan M, Heim MH \& Stoffel M 2011 MicroRNAs 103 and 107 regulate 
insulin sensitivity. Nature 474 649-653. (doi:10.1038/ nature10112)

Vanhoutte P, Nissen JL, Brugg B, Gaspera BD, Besson MJ, Hipskind RA \& Caboche J 2001 Opposing roles of Elk-1 and its brain-specific isoform, short Elk-1, in nerve growth factor-induced PC12 differentiation. Journal of Biological Chemistry 276 5189-5196. (doi:10.1074/jbc.M006678200)

Wang Q, Li YC, Wang J, Kong J, Qi Y, Quigg RJ \& Li X 2008 miR-17-92 cluster accelerates adipocyte differentiation by negatively regulating tumor-suppressor Rb2/p130. PNAS 105 2889-2894. (doi:10.1073/ pnas.0800178105)

Wang W, Huang L, Huang Y, Yin JW, Berk AJ, Friedman JM \& Wang G 2009 Mediator MED23 links insulin signaling to the adipogenesis transcription cascade. Developmental Cell 16 764-771. (doi:10.1016/j. devcel.2009.04.006)
Xie H, Lim B \& Lodish HF 2009 MicroRNAs induced during adipogenesis that accelerate fat cell development are downregulated in obesity. Diabetes 58 1050-1057. (doi:10.2337/ db08-1299)

Yang SH, Shore P, Willingham N, Lakey JH \& Sharrocks AD 1999 The mechanism of phosphorylation-inducible activation of the ETSdomain transcription factor Elk-1. EMBO Journal 18 5666-5674. (doi:10.1093/emboj/18.20.5666)

Yeh WC, Cao Z, Classon M \& McKnight SL 1995 Cascade regulation of terminal adipocyte differentiation by three members of the C/EBP family of leucine zipper proteins. Genes \& Development 9 168-181. (doi:10.1101/gad.9.2.168)

Yordy JS \& Muise-Helmericks RC 2000 Signal transduction and the Ets family of transcription factors. Oncogene 19 6503-6513. (doi:10.1038/sj.onc.1204036)

Received in final form 7 March 2016

Accepted 5 April 2016

Accepted Preprint published online 6 May 2016
Published by Bioscientifica Ltd. 